\title{
Multiple Mediastinal Arteriovenous Fistulas in a Patient with Myocardial Ischemia Symptoms and Tachy-Brady Arrhythmias
}

\author{
István Kovács ${ }^{1}$, Sebastian Condrea ${ }^{2}$, Ioana Rodean ${ }^{2}$, Daniel Cernica ${ }^{2}$ \\ ${ }^{1}$ University of Medicine and Pharmacy, Tîrgu Mureș, Romania \\ ${ }^{2}$ Center of Advanced Research in Multimodality Cardiac Imaging, Cardio Med Medical Center, Tîrgu Mureș, Romania
}

\begin{abstract}
Arteriovenous fistulas are abnormal vascular communications between the arterial and venous system and may lead to the development of arteriovenous shunts. These malformations are extremely rare, and their diagnosis can be difficult due to the lack of symptoms or the absence of hemodynamic consequences. In many cases, arteriovenous fistulas are discovered by chance. In this brief report, we present the case of a 31-year-old male with symptoms suggestive of angina pectoris, tachycardia-bradycardia syndrome and multiple episodes of syncope. Multi-slice computed tomography revealed the presence of multiple mediastinal fistulas connecting the aorta, superior vena cava, pulmonary veins and azygos system.
\end{abstract}

Keywords: arteriovenous fistulas, CT angiography, aorta, superior vena cava, pulmonary veins, azygos system

\section{ARTICLE HISTORY}

Received: 5 February, 2017

Accepted: 13 March, 2017

\section{CORRESPONDENCE}

Ioana Rodean

Str. 22 Decembrie 1989 nr. 76

540124 Tîrgu Mureș, Romania

Tel: +40 265217333

E-mail: ioana_patricia91@yahoo.com

\section{INTRODUCTION}

Mediastinal vascular fistulas are the consequence of the abnormal variation of blood flow from one vessel to another and might develop between any arterial or venous structure. ${ }^{1-3}$ Nowadays, arteriovenous fistulas are more frequently diagnosed, even in asymptomatic patients, during routine investigations, due to the improvement of imaging techniques. ${ }^{4}$ Computed tomographic angiography (CTA) and conventional angiography are the goldstandard methods for diagnosing AV fistulas, being able to expose the convoluted pathways arising from abnormally connected vessels. ${ }^{1}$
Congenital and acquired fistulas connecting the arterial and venous systems are exceedingly rare. Clinical status and physical examination may vary from an asymptomatic pattern to audible thoracic murmur, dyspnea, or cardiac insufficiency. ${ }^{1,5}$

The case of a young man presenting with symptoms of myocardial ischemia but with no invasive or noninvasive evidence of any heart disease is discussed in this paper. Thoracic CT angiography showed multiple mediastinal tortuous pathways between the descending aorta and the superior vena cava, the aorta and the left superior pulmonary vein, and the azygos vein and the right superior pulmonary vein. 


\section{CASE REPORT}

The case concerns a 31-year-old man, with no history of cardiovascular disease, admitted to the Cardio Med Medical Center in Tîrgu Mureș, Romania.

He complained of anterior constrictive chest pain and tightness and rapid palpitations, followed by episodes of bradycardia associated with dizziness, syncope and shortness of breath.

The patient gave informed consent allowing the publication of his data, and the institution where the patient had been admitted approved the publication of the case.

The patient's clinical signs and symptoms were suggestive of a tachycardia-bradycardia type arrhythmia, but initial investigations - physical examination and laboratory tests - showed no significant findings.

An electrocardiogram (ECG) performed during chest pain showed normal sinus rhythm with a frequency of 70 beats per minute (bpm) and negative T waves in DIII and V1-V4. Resting ECG also showed normal sinus rhythm with a frequency of $75 \mathrm{bpm}$ and normal axis, but the $\mathrm{T}$ wave appeared to be flat in DIII and $\mathrm{aVF}$, and negative $\mathrm{T}$ waves were seen in V1 and V2.

Echocardiography parameters were within normal ranges. However, the posterior and lateral pericardium appeared to have a higher echodensity than normal.

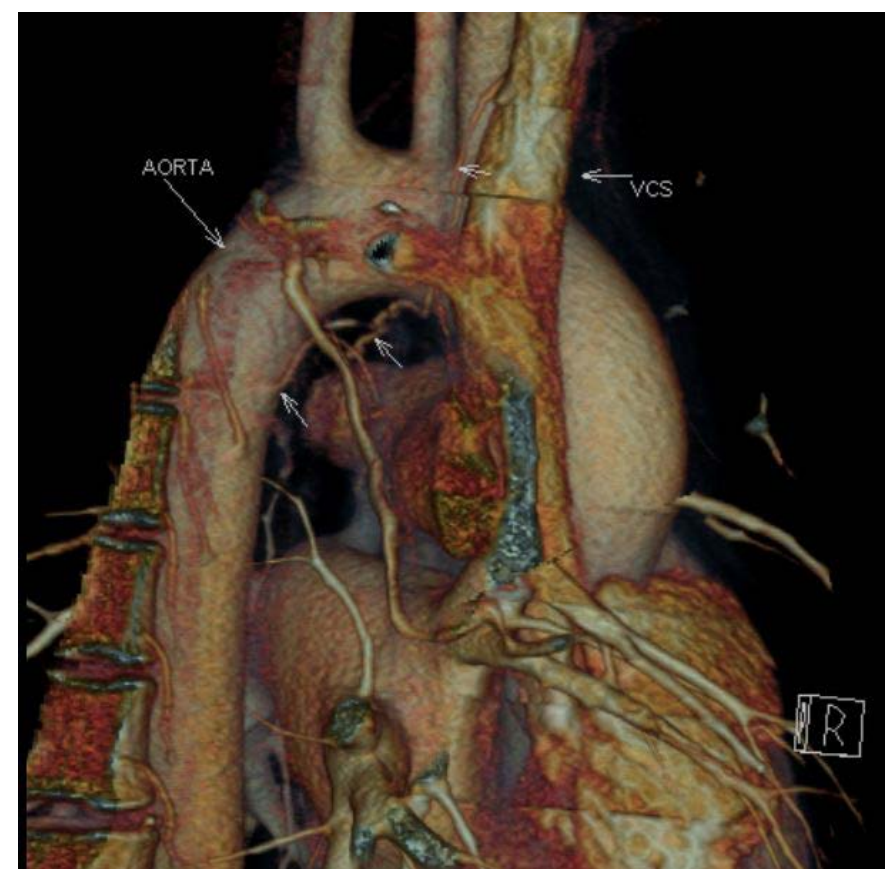

FIGURE 1. CTA showing arterial-venous fistula between azygos vein and right superior pulmonary vein; aorta and superior vena cava; descending aorta and left superior pulmonary vein
The patient also underwent cycle ergometer stress testing, which was positive for the alteration of myocardial perfusion. The test was stopped after 2 minutes (100 W) due to the dyspnea and dizziness, though there were no signs of any angina symptoms. During the cycle ergometer stress testing, the ECG showed $\mathrm{T}$ wave variations that became negative in DII, DIII, aVF, and V2-V5. After a twominute resting period, the patient's ECG returned to normal, with positive $\mathrm{T}$ waves in these leads.

Taking into consideration the palpitation symptoms, a Holter ECG was performed, which denoted three tachycardia events, with a maximum heart rate of $110 \mathrm{bpm}$, alternating with bradycardia episodes with a minimum heart rate of $30-40 \mathrm{bpm}$.

In order to assess the presence of possible coronary lesions, a CTA was performed, starting with the dynamic administration of $100 \mathrm{ml}$ of non-ionic contrast material Iopamiro 370 (Patheon Italy SpA, Italy) at a rate of $5 \mathrm{ml} / \mathrm{s}$, followed by $100 \mathrm{ml}$ saline solution at $5 \mathrm{ml} / \mathrm{s}$, via the right anterior cubital approach, followed by CT scanning using a Somatom Sensation 64 slices CT (Siemens, Erlangen, Germany). The examination was well tolerated, and there were no complications.

Three-dimensional post-processing programs - MPR, MIP, and reconstruction curved VRT - were used, and the examination showed a dominance of the right coronary

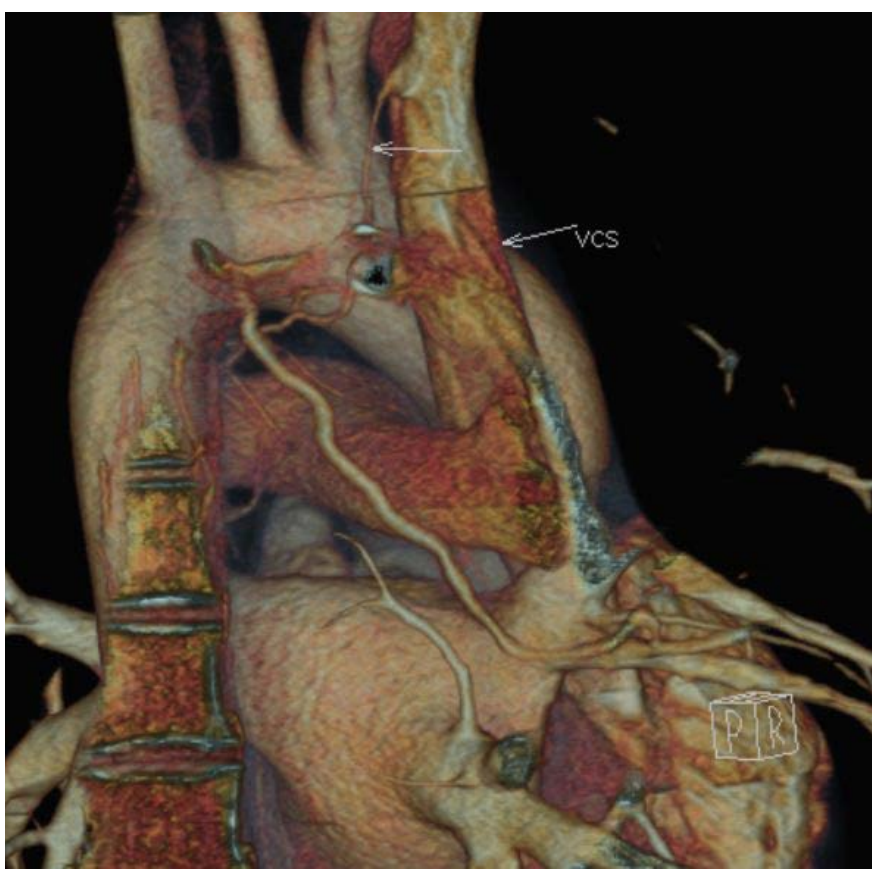

FIGURE 2. CTA showing arterial-venous fistula between descending aorta and left superior pulmonary vein; aorta and superior vena cava 


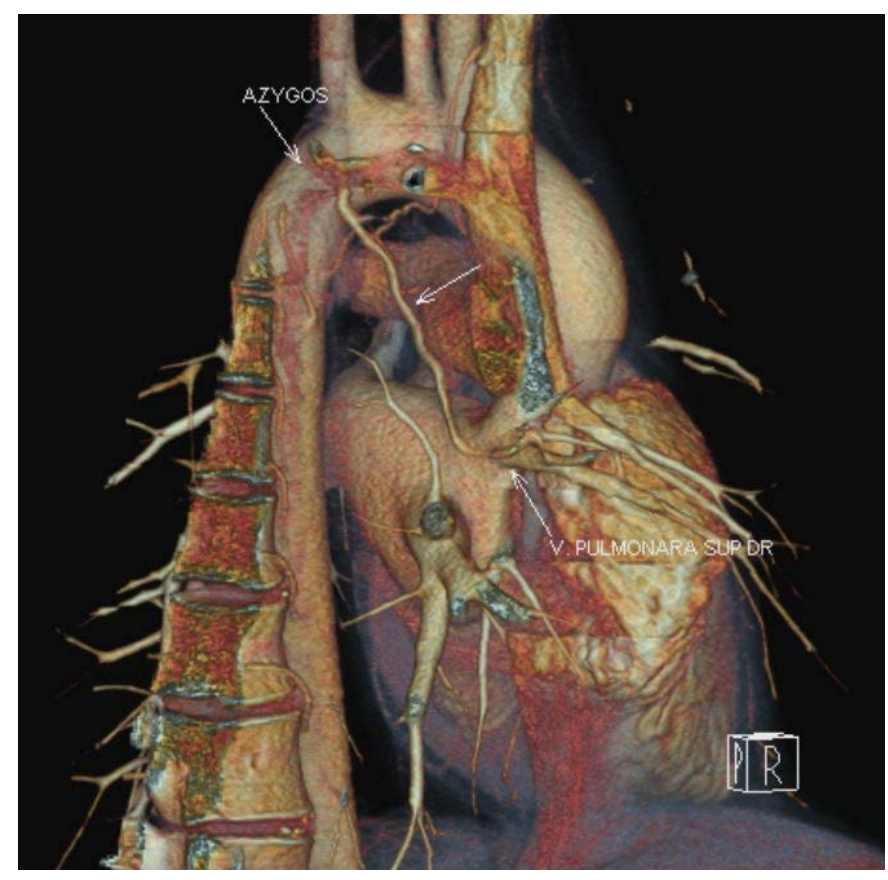

FIGURE 3. CTA showing fistula between azygos vein and superior right pulmonary vein

artery, with the coronary arteries, pericardium, thoracic aorta, lungs, and pleura showing normal characteristics. Due to these normal results and the patient's subjective complaints of angina and palpitations, the investigation was extended, and the thoracic vessels were investigated in more detail. Following CT angiography, a diagnosis of $\mathrm{AV}$ fistulas located in the middle mediastinum was established. Small diameter tortuous vascular pathways interposed between the descending aorta and superior vena cava (Figure 1), the aorta and left superior pulmonary vein (Figures 2 and 3), and between the azygos and right superior pulmonary vein were identified (Figure 4).

The surgical team recommended the ligature of the venous fistulas between the descending aorta and superior vena cava, the aorta and left superior pulmonary vein, and the azygos and right superior pulmonary vein, but the patient refused the intervention.

During hospitalization, the patient was treated with anti-ischemic therapy, $25 \mathrm{mg}$ of beta blocker (metoprolol $25 \mathrm{mg}$, two times per day, for an indefinite period, but with regular check-ups and re-assessment of treatment), with significant improvement of his symptoms, in the absence of surgical intervention.

\section{DISCUSSION}

Arteriovenous fistulas are extremely rare, being described in only a few cases in the literature. ${ }^{1}$ Due to the similar-

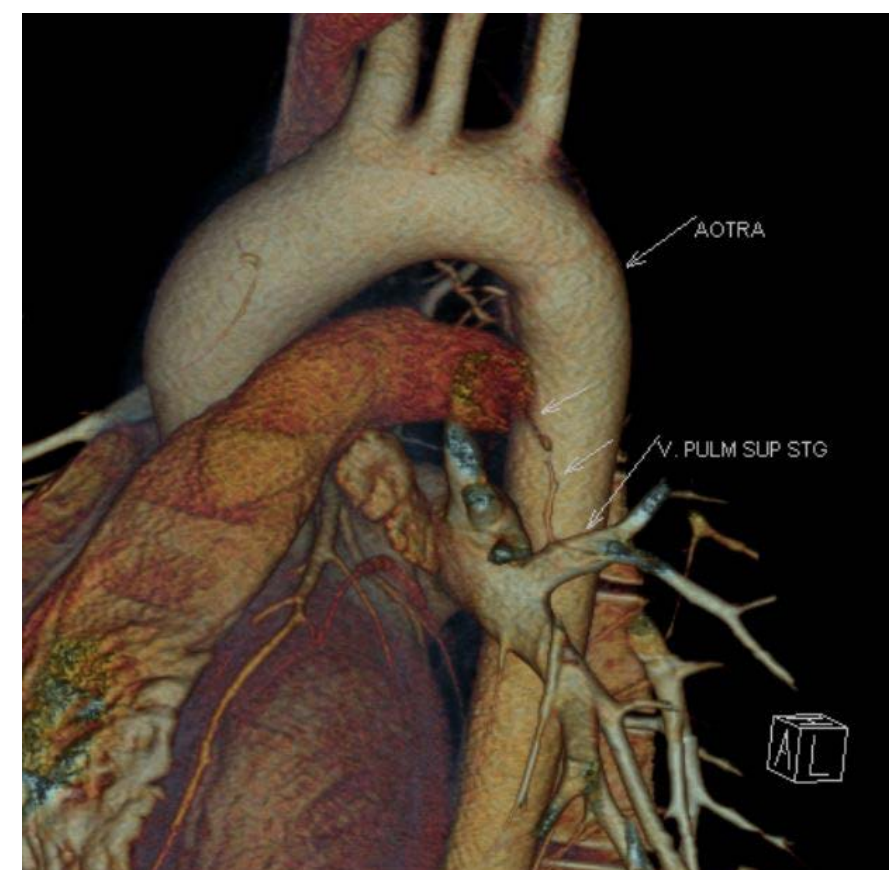

FIGURE 4. CTA showing fistula between the upper aorta and left superior pulmonary vein

ity of cardiac and extra-cardiac symptoms associated with this condition, the diagnosis of these cases may be problematic. ${ }^{6}$

Soler et al. reported that an AV fistula originating from the descending aorta and draining into the azygos vein is a very rare condition. ${ }^{6}$ They attributed this malformation to abnormal connections between embryologic arterial and venous channels. The signs and symptoms associated with the presence of an AV fistula depend largely on the size of the fistula and the degree of resulting left-to-right shunting.7 Several studies have suggested that the malformations of the great vessels and abnormal drainage can produce arrhythmias in patients with different types of $\mathrm{AV}$ fistula. ${ }^{8}$

The noninvasive diagnosis of this disease is based on thoracic multi-slice computed tomography, CT angiography, conventional angiography, magnetic resonance imaging, and radionuclide angiograms., ${ }^{1,9}$

Although in the past open surgical correction was the gold-standard of treatment of this disease, nowadays interventional treatment is preferred, including balloon occlusion, coiling embolization, and other occlusion devices such as the Gianturco coil or, more recently, the Gianturco-Grifka Vascular Occlusion Device (Cook Inc.). ${ }^{10-12}$ Despite all new therapeutic techniques, the treatment procedure depends on the origin of the AV fistula. Large congenital fistulas may require surgical intervention. However, small ones may be treated conservatively. ${ }^{1}$ 
The case reported here is the first documented case of multiple mediastinal arterial-veno-venous fistulas, which was associated with a suggestive ECG pattern including negative $\mathrm{T}$ waves and positive cycle ergometer stress test. Moreover, another particularity of this case is that the patient was admitted several times because of his chest pain accompanied by tachy-brady arrhythmia and episodes of syncope, without any precise etiology. On his last admission with these symptoms, the decision was taken to perform a CTA, which revealed no evidence of coronary or pulmonary abnormalities. Additionally, CT angiography investigations revealed multiple small caliber fistulas in the mediastinum, between the descending aorta and the superior vena cava, the aorta and the left superior pulmonary vein, and the azygos vein and the right superior pulmonary vein.

\section{CONCLUSION}

Thoracic arteriovenous fistulas are extremely rare malformations that are usually underdiagnosed due to equivalent signs and symptoms of other cardio-pulmonary and mediastinal conditions. Based on the complexity and the type of the shunt, the symptoms can vary from asymptomatic conditions to heart failure. Because of the lack of symptoms, in many cases, the diagnosis is made by chance. Hemodynamic consequences can be absent in small diameter shunts or can be substantial in large ones.

CTA, the gold-standard noninvasive imaging technique used for the assessment of these cases, linked with complex 3D post-processing techniques, can reveal the location of the malformations, assess their complexity and help guide treatment planning.

\section{CONFLICT OF INTEREST}

Nothing to declare.

\section{REFERENCES}

1. Martinez-Jimenez S, Heyneman LE, McAdams HP, et al. Nonsurgical Extracardiac Vascular Shunts in the Thorax: Clinical and Imaging Characteristics. RadioGraphics. 2010;10.1148/rg.e41.

2. Martinez-Jimenez S, Heyneman LE, McAdams HP, et al. The science and practice of pediatric cardiology. Radiographics. 2010;30:e41. doi: 10.1148/rg.e41.

3. Sapire DW, Lobe TE, Swischuk LE, Casta A, Schwartz MZ, Droge M. Subclavian-artery-to-innominate-vein fistula presenting with congestive failure in a newborn infant. Pediatr Cardiol. 1983;4:155-157.

4. Ghandour A, Partovi S, Karuppasamy K, Rajiah P. Congenital anomalies of the IVC - embryological perspectives and clinical relevance. Cardiovasc Diagn Ther. 2016;6:482-492. doi: 10.21037/cdt.2016.11.18.

5. Romero FD, Fernández EM, Albelo TP, González HV, Gonzalez IA. Congenital aorto-azygous fistula treated with coil embolization: case report and review of the literature. Cardiovasc Intervent Radiol. 2006;29:907-910. doi: 10.1007/ s00270-005-0163-4.

6. Soler P, Mehta AV, Garcia OL, Kaiser G, Tamer D. Congenital systemic arteriovenous fistula between the descending aorta, azygos vein, and superior vena cava. Chest. 1981;80:647-649.

7. Recto MR, Elbl F. Transcatheter Coil Occlusion of a Thoracic Arteriovenous Fistula in an Infant with Congestive Heart Failure. Tex Heart Inst J. 2001;28:119-121.

8. Doig JC, Saito J, Harris L, Downar E. Coronary sinus morphology in patients with atrioventricular junctional reentry tachycardia and other supraventricular tachyarrhythmias. Circulation. 1995;92:436-441.

9. Benson R, Songrug T. CT appearance of persistent left superior vena cava, anomalous right superior pulmonary venous return into the right sided superior vena cava and a sinus venosustype atrial septal defect. Br J Radiol. 2009;82:e235-e239. doi: $10.1259 /$ bjr/27663006.

10. Anderson JH, Wallace S, Gianturco C. Transcatheter intravascular coil occlusion of experimental arteriovenous fistulas. AJR Am J Roentgenol. 1977;129:795-798.

11. Bricelj V, Robida A. Coil occlusion of arteriovenous fistula of the vertebral artery in a child. Cathet Cardiovasc Diagn. 1998;43:434-437.

12. Ebeid MR, Braden DS, Gaymes CH, Joransen JA. Closure of a large pulmonary arteriovenous malformation using multiple Gianturco-Grifka vascular occlusion devices. Cathet Cardiovasc Interv. 2000;49:426-429. 